CARPATHIAN J. MATH.

Volume 37 (2021), No. 2,

Pages 259 - 263
Online version at https : //www . carpathian. cunbm . utcluj. ro/

Print Edition: ISSN 1584 - 2851; Online Edition: ISSN 1843 - 4401

DOI: https://doi.org/10.37193/CJM.2021.02.11

Dedicated to Prof. Ioan A. Rus on the occasion of his $85^{\text {th }}$ anniversary

\title{
Application of a fixed point theorem on infinite cartesian product to an infinite system of differential equations
}

\author{
MARCEL-ADRIAN ŞERBAN
}

\begin{abstract}
In the paper Operators on infinite dimensional cartesian product, (Analele Univ. Vest Timişoara, Mat. Inform., 48 (2010), 253-263), by I. A. Rus and M. A. Şerban, the authors give a generalization of the Fibre contraction theorem on infinite dimensional cartesian product. In this paper we give an application of this abstract result to an infinite system of differential equations.
\end{abstract}

\section{INTRODUCTION}

For a triangular operator $A: X \times Y \rightarrow X \times Y, A=(B, C)$, where $B: X \rightarrow X$ and $C: X \times Y \rightarrow Y$ we can formulate the following problem:

Problem 1.1 (Fibre Picard operator problem). Let $(X, \stackrel{1}{\rightarrow})$ and $(Y, \stackrel{2}{\rightarrow})$ be two L-spaces.

Let $B: X \rightarrow X$ be a WPO and $C: X \times Y \rightarrow Y$ be such that $C(x, \cdot): Y \rightarrow Y$ is a WPO for every $x \in X$.

In which conditions the operator $A=(B, C)$ is a WPO?

Results concerning this problem in different settings were obtained by M. W. Hirsch, C. C. Pugh [5], J. Sotomayor [16], I. A. Rus [6], [7], [8], S. Andrász [1], C. Bacoţiu [2], I. A. Rus and M. A. Şerban [13], [15], M. A. Şerban [17], [18], [19], [20].

In the paper I. A. Rus and M. A. Şerban [14], the authors give a generalization of the Fibre contraction theorem for triangular operators defined on infinite dimensional cartesian product spaces. The aim of this paper is to present an application of this abstract result to an infinite system of differential equations.

\section{PRELIMINARIES}

In this paper we shall use the terminologies and notations from [10] and [11]. For the convenience of the reader we shall recall some of them.

Let $(X, \rightarrow)$ be an L-space and $f: X \rightarrow X$ an operator. We denote by $f^{0}:=1_{X}, f^{1}:=f$, $f^{n+1}:=f \circ f^{n}, n \in \mathbb{N}$ the iterate operators of the operator $A$. Also:

$$
\begin{gathered}
P(X):=\{Y \subseteq X \mid Y \neq \emptyset\} \\
F_{f}:=\{x \in X \mid f(x)=x\}
\end{gathered}
$$

By $(X, \rightarrow)$ we will denote an $L$-space. Actually, an $L$-space is any set endowed with a structure implying a notion of convergence for sequences (for examples of $L$-spaces see Fréchet [4], Blumenthal [3] and I. A. Rus [10]).

Let $(X, \rightarrow)$ be an $L$-space.

Received: 26.01.2021. In revised form: 19.04.2021. Accepted: 22.04.2021

2010 Mathematics Subject Classification. 47H10, 54H25, 45G15.

Key words and phrases. Weakly Picard operators, Picard operators, triangular operators, fibre contraction principle, infinite system of differential equations. 
Definition 2.1. $f: X \rightarrow X$ is called a Picard operator (briefly PO) if:

(i) $F_{f}=\left\{x^{*}\right\}$;

(ii) $f^{n}(x) \rightarrow x^{*}$ as $n \rightarrow \infty$, for all $x \in X$.

Definition 2.2. $f: X \rightarrow X$ is said to be a weakly Picard operator (briefly WPO) if the sequence $\left(f^{n}(x)\right)_{n \in N}$ converges for all $x \in X$ and the limit (which may depend on $x$ ) is a fixed point of $f$.

If $f: X \rightarrow X$ is a WPO, then we may define the operator $f^{\infty}: X \rightarrow X$ by

$$
f^{\infty}(x):=\lim _{n \rightarrow \infty} f^{n}(x) .
$$

Obviously $f^{\infty}(X)=F_{f}$. Moreover, if $f$ is a $\mathrm{PO}$ and we denote by $x^{*}$ its unique fixed point, then $f^{\infty}(x)=x^{*}$, for each $x \in X$.

For a triangular operator $A: X \times Y \rightarrow X \times Y, A=(B, C)$, where $B: X \rightarrow X$ and $C: X \times Y \rightarrow Y$ we have the following result:

Theorem 2.1 (Fibre contraction principle). ([5], [7], [8], [16]) We suppose that:

(i) $\left(Y, d_{Y}\right)$ is a complete metric space;

(ii) $B$ is a WPO;

(iii) $C(x, \cdot): Y \rightarrow Y$ is $\alpha$-contraction for every $x \in X$;

(iv) $C: X \times Y \rightarrow Y$ is continuous.

Then

(a) $A$ is a WPO;

(b) If $B$ is a $P O$ then $A$ is a $P O$.

Theorem 2.1 was extended in [14] to the case of triangular operators defined on infinite dimensional cartesian product spaces in the following way.

Let $\left(X_{i}, d_{i}\right), i \in \mathbb{N}^{*}$, be metric spaces and $\left(X_{0}, \rightarrow\right)$ an L-space and we denote by $X=$ $\prod_{i \in \mathbb{N}} X_{i}$, the cartesian product of $X_{i}, i \in \mathbb{N}$. We organize $X=\prod_{i \in \mathbb{N}} X_{i}$ as an L-space by termwise convergence, i.e.

$$
x^{k} \stackrel{t}{\rightarrow} x, \text { as } k \rightarrow+\infty \Leftrightarrow\left\{\begin{array}{l}
x_{0}^{k} \rightarrow x_{0} \text {, as } k \rightarrow+\infty, \\
x_{n}^{k} \stackrel{d_{n}}{\rightarrow} \text { as } k \rightarrow+\infty, \forall n \in \mathbb{N}^{*} .
\end{array}\right.
$$

We consider the operators $A_{k}: X_{0} \times X_{1} \times \ldots \times X_{k} \rightarrow X_{k}, k \in \mathbb{N}$, and

$$
\begin{gathered}
A: X \rightarrow X \\
A\left(x_{0}, x_{1}, \ldots, x_{n}, \ldots\right)=\left(A_{0}\left(x_{0}\right), A_{1}\left(x_{0}, x_{1}\right), \ldots, A_{n}\left(x_{0}, x_{1}, \ldots, x_{n}\right), \ldots\right)
\end{gathered}
$$

Theorem 2.2. [14] Suppose that:

(i) $\left(X_{k}, d_{k}\right)$ is a complete metric space for every $k \in \mathbb{N}^{*}$;

(ii) $A_{0}$ is WPO;

(iii) $A_{k}\left(x_{0}, x_{1}, \ldots, x_{k-1}, \cdot\right): X_{k} \rightarrow X_{k}$ is $\alpha_{k}$-contraction, for every $k \in \mathbb{N}^{*}$;

(iv) $A$ is continuous.

Then $A$ is WPO. Moreover, if $A_{0}$ is PO then $A$ is PO.

\section{CAUCHY PROBLEM FOR A SYSTEM OF INFINITE DIFFERENTIAL EQUATIONS}

We consider the Cauchy problem:

$$
\left\{\begin{aligned}
x_{i}^{\prime}(t) & =f_{i}\left(t, x_{1}(t), \ldots, x_{m}(t)\right), & & t \in[a ; b], i=\overline{1, m} \\
x_{m+k}^{\prime}(t) & =f_{m+k}\left(t, x_{1}(t), \ldots, x_{m+k}(t)\right), & & t \in[a ; b], k \in \mathbb{N}^{*} \\
x(a) & =x^{0} & &
\end{aligned}\right.
$$



where $x=\left(x_{1}, x_{2}, \ldots\right)$ and $x^{0}=\left(x_{1}^{0}, x_{2}^{0}, \ldots\right) \in \mathbb{R}^{\infty}$.

The Cauchy problem (3.2) is equivalent with the following infinite system of integral equations:

$$
\left\{\begin{array}{c}
\left(\begin{array}{c}
x_{1}(t) \\
\vdots \\
x_{m}(t)
\end{array}\right)=\left(\begin{array}{c}
x_{1}^{0} \\
\vdots \\
x_{m}^{0}
\end{array}\right)+\left(\begin{array}{c}
\int_{a}^{t} f_{1}\left(s, x_{1}(s), \ldots, x_{m}(s)\right) d s \\
\vdots \\
\int_{a}^{t} f_{m}\left(s, x_{1}(s), \ldots, x_{m}(s)\right) d s
\end{array}\right), \\
x_{m+k}(t)=x_{m+k}^{0}+\int_{a}^{t} f_{m+k}\left(s, x_{1}(s), \ldots, x_{m+k}(s)\right) d s, k \in \mathbb{N}^{*}, t \in[a ; b] .
\end{array}\right.
$$

We consider the Banach spaces $X_{0}=\left(C\left([a, b], \mathbb{R}^{m}\right),\|\cdot\|_{\tau_{0}}\right), X_{k}=\left(C[a, b],\|\cdot\|_{\tau_{k}}\right), k \in$ $\mathbb{N}^{*}$, where $\|\cdot\|_{\tau_{k}}$ are the corresponding Bielecki norms:

$$
\begin{gathered}
\|y\|_{\tau_{0}}=\max _{i=\overline{1, m}} \max _{t \in[a, b]}\left\{\left|y_{i}(t)\right| \cdot e^{-\tau_{0}(t-a)}\right\}, y \in C\left([a, b], \mathbb{R}^{m}\right), \\
\|z\|_{\tau_{k}}=\max _{t \in[a, b]}\left\{|z(t)| \cdot e^{-\tau_{k}(t-a)}\right\}, z \in C[a, b], k \in \mathbb{N}^{*},
\end{gathered}
$$

and the operators $A_{0}: X_{0} \rightarrow X_{0}$,

$$
A_{0}\left(x_{1}, \ldots, x_{m}\right)(t)=\left(\begin{array}{c}
x_{1}^{0} \\
\vdots \\
x_{m}^{0}
\end{array}\right)+\left(\begin{array}{c}
\int_{a}^{t} f_{1}\left(s, x_{1}(s), \ldots, x_{m}(s)\right) d s \\
\vdots \\
\int_{a}^{t} f_{m}\left(s, x_{1}(s), \ldots, x_{m}(s)\right) d s
\end{array}\right),
$$

and $A_{k}: X_{0} \times X_{1} \times \ldots \times X_{k} \rightarrow X_{k}, k \in \mathbb{N}^{*}$,

$$
A_{k}\left(x_{1}, \ldots, x_{m+k}\right)(t)=x_{m+k}^{0}+\int_{a}^{t} f_{m+k}\left(s, x_{1}(s), \ldots, x_{m+k}(s)\right) d s .
$$

It is clear that for operator $A: X \rightarrow X$, where $X=\prod_{i \in \mathbb{N}} X_{i}$ and $A=\left(A_{0}, A_{1}, \ldots, A_{k}, \ldots\right)$, the infinite system of integral equations (3.3) is equivalent with fixed point equation in $X$ :

$$
x=A(x) \text {. }
$$

We have the following result:

Theorem 3.3. We consider the Cauchy problem (3.2) and we suppose that:

(i) $f_{i}$ are continuous functions, $i \in \mathbb{N}^{*}$;

(ii) there exists $L_{0}>0$ such that

$$
\left|f_{i}\left(t, u_{1}, \ldots, u_{m}\right)-f_{i}\left(t, v_{1}, \ldots, v_{m}\right)\right| \leq L_{0} \sum_{j=1}^{m}\left|u_{j}-v_{j}\right|,
$$

for all $t \in[a ; b], u_{1}, \ldots, u_{m}, v_{1}, \ldots, v_{m} \in \mathbb{R}^{m}, i=\overline{1, m}$;

(iii) there exist $L_{k}>0, k \in \mathbb{N}^{*}$, such that:

$\left|f_{m+k}\left(t, u_{1}, \ldots, u_{m+k-1}, v_{1}\right)-f_{m+k}\left(t, u_{1}, \ldots, u_{m+k-1}, v_{2}\right)\right| \leq L_{k}\left|v_{1}-v_{2}\right|$, for all $t \in[a ; b], u_{j} \in \mathbb{R}, j=\overline{1, m+k-1}, v_{1}, v_{2} \in \mathbb{R}$.

Then the Cauchy problem (3.2) has a unique solution in $X$. 
Proof. Condition (i) ensures the well definition of the operators $A_{0}, A_{1}, \ldots, A_{k}, \ldots$, and the continuity of the operator $A$. From condition (ii) we get

$$
\left\|A_{0}(\mathbf{x})-A_{0}(\mathbf{y})\right\|_{\tau_{0}} \leq \frac{L_{0}}{\tau_{0}}\|\mathbf{x}-\mathbf{y}\|_{\tau_{0}},
$$

for every $\mathbf{x}=\left(x_{1}, \ldots, x_{m}\right), \mathbf{y}=\left(y_{1}, \ldots, y_{m}\right) \in X_{0}$. If we choose $\tau_{0}>0$ such that $\frac{L_{0}}{\tau_{0}}<1$, (for example $\tau_{0}=L_{0}+1$ ), then $A_{0}$ is an $\alpha_{0}$ - contraction, with $\alpha_{0}=\frac{L_{0}}{\tau_{0}}$, therefore $A_{0}$ is PO.

Also, for $k \in \mathbb{N}^{*}$, we have

$$
\left\|A_{k}\left(x_{0}, \ldots, x_{m+k-1}, y_{1}\right)-A_{k}\left(x_{0}, \ldots, x_{m+k-1}, y_{2}\right)\right\|_{\tau_{k}} \leq \frac{L_{k}}{\tau_{k}}\left\|y_{1}-y_{2}\right\|_{\tau_{k}}
$$

for every $\left(x_{0}, \ldots, x_{m+k-1}\right) \in X_{0} \times \ldots \times X_{k-1}$ and $y_{1}, y_{2} \in X_{k}$, which shows that $A_{k}\left(x_{0}, \ldots, x_{m+k-1}, \cdot\right): X_{k} \rightarrow X_{k}$ is an $\alpha_{k}$-contraction, for a suitable choice of $\tau_{k}>0$, (for example $\tau_{k}=L_{k}+1$ ), thus we apply Theorem 2.2 and we obtain that $A: X \rightarrow X$ is PO, therefore the system (3.3) has a unique solution in $X$, so the Cauchy problem (3.2) has a unique solution.

Example 3.1. Let consider the Cauchy problem:

$$
\left\{\begin{aligned}
x_{i}^{\prime}(t) & =g_{i}\left(t, x_{1}(t), \ldots, x_{m}(t)\right), & & t \in[a ; b], i=\overline{1, m} \\
x_{m+k}^{\prime}(t) & =g_{m+k}\left(t, x_{1}(t), \ldots, x_{m+k-1}(t)\right)+k \cos \left(x_{m+k}\right), & & t \in[a ; b], k \in \mathbb{N}^{*} \\
x(a) & =x^{0} & &
\end{aligned}\right.
$$

$x^{0} \in \mathbb{R}^{\infty}$. We suppose that:

(i) $g_{i}$ are continuous functions, $i \in \mathbb{N}^{*}$;

(ii) there exists $L_{0}>0$ such that

$$
\left|g_{i}\left(t, u_{1}, \ldots, u_{m}\right)-g_{i}\left(t, v_{1}, \ldots, v_{m}\right)\right| \leq L_{0} \sum_{j=1}^{m}\left|u_{j}-v_{j}\right|,
$$

for all $t \in[a ; b], u_{1}, \ldots, u_{m}, v_{1}, \ldots, v_{m} \in \mathbb{R}^{m}, i=\overline{1, m}$.

Then the Cauchy problem (3.6) has a unique solution.

Proof. We apply Theorem 3.3 for $f_{i}, i \in \mathbb{N}^{*}$,

$$
\begin{gathered}
f_{i}\left(t, u_{1}, \ldots, u_{m}\right)=g_{i}\left(t, u_{1}, \ldots, u_{m}\right), i=\overline{1, m}, \\
f_{m+k}\left(t, u_{1}, \ldots, u_{m+k-1}, u_{m+k}\right)=g_{m+k}\left(t, u_{1}, \ldots, u_{m+k-1}\right)+k \cos \left(u_{m+k}\right), k \in \mathbb{N}^{*} .
\end{gathered}
$$

It is easy to see that $f_{i}$ is lipschitz with constant $L_{0}, i=\overline{1, m}$, and $f_{m+k}\left(t, u_{1}, \ldots, u_{m+k-1}, \cdot\right)$ is lipschitz with $L_{k}=k, k \in \mathbb{N}^{*}$, therefore all the condition from Theorem 3.3 are satisfied, so we get the conclusion.

\section{REFERENCES}

[1] Andrász, S., Fibre $\varphi$ - contraction on generalized metric spaces and applications, Mathematica, 45 (68)(2003), No. $1,3-8$

[2] Bacoţiu, C., Fibre Picard operators on generalized metric spaces, Sem. on Fixed Point Theory Cluj-Napoca, 1 (2000), 5-8

[3] Blumenthal, L. M., Theory and applications of distance geometry, Oxford University Press, 1953

[4] Fréchet, M., Les espaces abstraits, Gauthier-Villars, Paris, 1928

[5] Hirsch, M. W. and Pugh, C. C., Stable manifolds and hyperbolic sets, Proc. Symp. in Pure Math., 14 (1970), A. M. S., 133-143

[6] Rus, I. A., A fibre generalized contraction theorem and applications, Mathematica, 41 (1999), No. 1, 85-90

[7] Rus, I. A., Fibre Picard operators and applications, Studia Univ. Babeş-Bolyai Math., 44 (1999), 89-98

[8] Rus, I. A., Fibre Picard operators on generalized metric spaces and applications, Scripta Sc. Math., 1 (1999), 326-334 
[9] Rus, I. A., Generalized Contractions and Applications, Cluj University Press, Cluj-Napoca, 2001

[10] Rus, I. A., Picard operators and applications, Sci. Math. Jpn., 58 (2003), 191-219

[11] Rus, I. A., Weakly Picard operators and applications, Seminar on Fixed Point Theory, Cluj-Napoca, 2 (2001), $41-58$

[12] Rus, I. A., Petruşel, A. and Petruşel, G., Fixed Point Theory, Cluj University Press, 2008

[13] Rus, I. A. and Şerban, M. A., Basic problems of the metric fixed point theory and the relevance of a metric fixed point theorem, Carpathian J. Math., 29 (2013), No. 2, 239-258

[14] Rus, I. A. and Şerban, M. A., Operators on infinite dimensional cartesian product, Analele Univ. Vest Timişoara, Mat. Inform., 48 (2010), 253-263

[15] Rus, I. A. and Şerban, M. A. Some generalizations of a Cauchy Lemma and Applications, in Topics in Mathematics, Computer Science and Philosophy, (Şt. Cobzaş Ed.), Cluj University Press, 2008, 173-181

[16] Sotomayor, J., Smooth dependence of solutions of differential equations on initial data: a simple proof, Bol. Soc. Bras. Mat., 4 (1973), 55-59

[17] Şerban, M. A., Fibre $\varphi$-contractions, Studia Univ. Babeş-Bolyai, Math., 44 (1999), No. 3, 99-108

[18] Şerban, M. A., Fibre contraction theorem in generalized metric spaces, Automat. Comput. Appl. Math., 16 (2007), No. 1, 139-144

[19] Şerban, M. A., The fixed point theory for the operators on cartesian product, (Romanian), Cluj University Press, Cluj-Napoca, 2002

[20] Şerban, M. A., Saturated fibre contraction principle, Fixed Point Theory, 18 (2017), No. 2, 729-740

DEPARTMENT OF MATHEMATICS

BABEŞ-BOLYAI UNIVERSITY OF CLUJ-NAPOCA

KOGĂLniceAnu 1, 400084 CluJ-NAPOCA, RoMANia

Email address: mserban@math.ubbcluj.ro, marcel.serban@ubbcluj.ro 